\title{
The Erlenmeyer synthesis with a thioazlactone
}

\author{
Sosale Chandrasekhar* and Malempati Srimannarayana \\ Department of Organic Chemistry, Indian Institute of Science, Bangalore 560 012, India \\ E-mail:sosale@orgchem.iisc.ernet.in
}

\begin{abstract}
2-Phenylthiazolin-5-one (2b) was generated in situ and condensed with various aldehydes in $\mathrm{CH}_{2} \mathrm{Cl}_{2}$ (r.t./20 min.), to obtain the corresponding 4-alkylidene or arylidene products in good yields (68-81\%). The reaction is catalyzed by basic lead acetate, and is equally successful with both aliphatic and aromatic aldehydes. This mild version of the classical Erlenmeyer azlactone synthesis is apparently enabled by the enhanced aromaticity of the thioazlactone anion intermediate.
\end{abstract}

Keywords: Amino acid, aromaticity, Erlenmeyer, lead acetate, thioazlactone

\section{Introduction}

We have recently reported interesting mechanistic and synthetic findings on the classical Erlenmyer azlactone synthesis, ${ }^{1}$ which remains a useful approach to the synthesis of $\alpha$-amino acids. ${ }^{2}$ In particular, we have shown that the reaction is apparently facilitated by the aromaticity of the intermediate azlactone anion that is generated under the basic reaction conditions. We have also reported a microwave method that extends the scope of the reaction to include a range of aliphatic aldehydes, thus removing a serious limitation to the classical protocol. We now report a useful extension of the classical synthesis by employing a thioazlactone analog, its anion being expected to be even more aromatically stabilized than the parent azlactone.

\section{Results and Discussion}

In the original Erlenmeyer synthesis, the azlactone (2a, Scheme 1) is generated in situ from the reaction of $N$-benzoylglycine (hippuric acid, 1a) with $\mathrm{Ac}_{2} \mathrm{O} / \mathrm{NaOAc}$ at elevated temperatures. In the key step, the azlactone anion (Ia) adds to an aromatic aldehyde (3), leading to the arylidene derivatives 4 . The success of the procedure apparently derives from the aromaticity of $\mathbf{I a},{ }^{1}$ and indicates that a thioazlactone (2b) would allow the reaction to occur under much milder 
conditions (in analogy to the greater aromaticity of thiophene relative to furan. ${ }^{3}$ ) Although this is indicated in previous reports on 2-phenylthiazolin-5-one, ${ }^{4,5}$ these did not attempt specifically to develop a milder variant of the Erlenmeyer synthesis. In our study, $N$-(thiobenzoyl)glycine (1b) was treated with $N, N^{\prime}$-dicyclohexylcarbodiimide (DCC) in $\mathrm{CH}_{2} \mathrm{Cl}_{2}$ at room temperature for 10 min. ${ }^{5-7}$ The reaction mixture was treated with an aldehyde (3) and a catalytic amount of basic lead acetate over 20 min. ${ }^{5,8,9}$ Minimal work-up and chromatographic purification led to the isolation of the 4-alkylidene or 4-arylidene thioazlactone derivative 5 , in generally excellent yields (Table 1).

These results indicate that the thiazolin-5-one (2b) generated in situ with DCC, ${ }^{7}$ has condensed with the aldehyde (3). Thus, the overall 'one-pot' process is reminiscent of the original Erlenmeyer reaction, although the present conditions are indeed exceedingly mild. The mechanism of the reaction most likely involves the initial activation of the carboxyl group of thiohippuric acid (1b) by DCC, followed by cyclization at the sulfur center to form $\mathbf{2} \mathbf{b}$. This is then apparently deprotonated by lead acetate to form anion $\mathbf{I b}$, which adds to the aldehyde $\mathbf{3}$ to furnish the intermediate alcohol IIb, which is dehydrated to form final product 5.

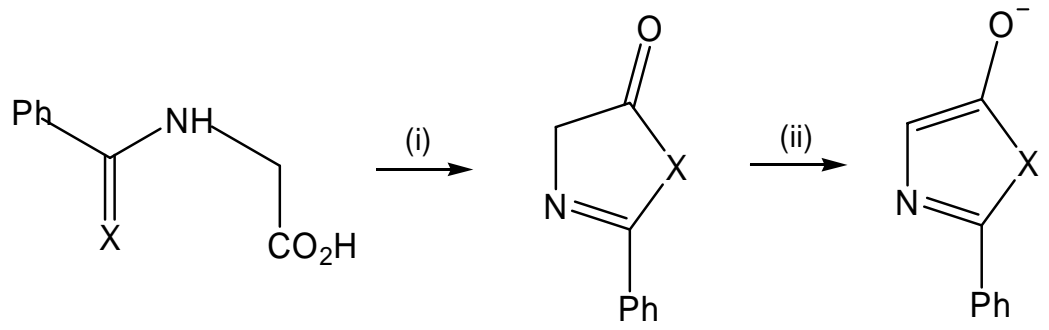

1a,b

2a,b

la,b

$\downarrow$ (iii)

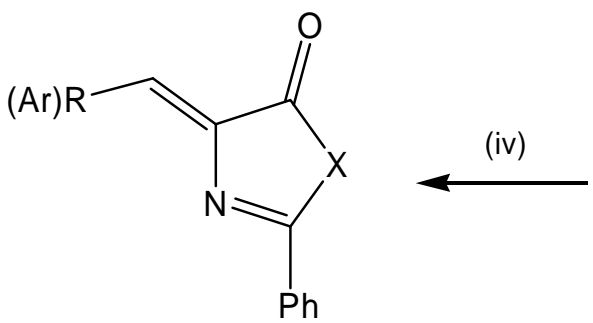

4,5

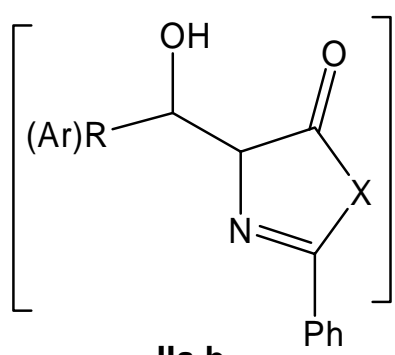

Ila,b

(i) $\mathrm{DCC} / \mathrm{CH}_{2} \mathrm{Cl}_{2} /$ r.t. (ii) $\mathrm{Pb}(\mathrm{OAC})_{2}-\mathrm{Pb}(\mathrm{OH})_{2}$ (0.05 equiv.).

(iii) $\mathrm{RCHO} / \mathrm{ArCHO}\left(3,1.2\right.$ equiv.); $\mathrm{H}^{+}$. (iv) $-\mathrm{H}_{2} \mathrm{O}$.

\begin{tabular}{|l|c|}
\hline Compound & $\mathrm{X}$ \\
\hline $1 \mathrm{a}, \mathbf{2 a}, \mathbf{I a}, \mathrm{Ila}, \mathbf{4}$ & $\mathrm{O}$ \\
\hline $\mathbf{1 b}, \mathbf{2 b}, \mathbf{I b}, \mathrm{Ilb}, \mathbf{5}$ & $\mathrm{S}$ \\
\hline
\end{tabular}

Scheme 1. The Erlenmeyer azlactone synthesis $(\mathbf{1 a} \rightarrow \mathbf{4})$ extended to the case of thioazlactone $2 b$. 
It is noteworthy that the condensation $(\mathbf{2 b}+\mathbf{3})$ occurs with a catalytic quantity of the exceedingly weak base, basic lead acetate $\left(\mathrm{Pb}(\mathrm{OAc})_{2} \cdot \mathrm{Pb}(\mathrm{OH})_{2}\right)^{5,8,9}$ This supports the case for the relatively enhanced aromaticity of Ib. The mild conditions are particularly suited for aliphatic aldehydes, several of which afford the expected products 5 in excellent yields (Table 1). The newly formed exocyclic double bond in $\mathbf{5}$ was assigned the $Z$ stereochemistry in analogy to the original azlactone synthesis. ${ }^{1}$

Table 1. Yields of 4-alkylidene or 4-arylidene thiazolin-5-ones 5 by the condensation of thioazlactone $\mathbf{4}$ with aldehydes $\mathbf{3}$ ( $c f$. Scheme 1)

\begin{tabular}{cccc}
\hline Entry & $\mathrm{R} / \mathrm{Ar}$ in $\mathbf{3}$ and $\mathbf{5}$ & Product $\mathbf{5}$ & Yield \% \\
\hline 1 & $\mathrm{Et}$ & $\mathbf{a}$ & 79 \\
2 & $n-\mathrm{Pr}$ & $\mathbf{b}$ & 81 \\
3 & $i$-Pr & $\mathbf{c}$ & 76 \\
4 & $i-\mathrm{Bu}$ & $\mathbf{d}$ & 73 \\
5 & $n-\mathrm{Hexyl}$ & $\mathbf{e}$ & 68 \\
6 & $\mathrm{Ph}$ & $\mathbf{f}$ & 70 \\
7 & $\mathrm{PhCH}=\mathrm{CH}-$ & $\mathbf{g}$ & 68 \\
8 & $4-\mathrm{MeO}-\mathrm{C}_{6} \mathrm{H}_{4^{-}}$ & $\mathbf{h}$ & 76 \\
\hline
\end{tabular}

\section{Conclusions}

In conclusion, we have developed an exceedingly mild version of the original Erlenmeyer azlactone synthesis that is equally applicable to aromatic and aliphatic aldehydes.

\section{Experimental Section}

General Procedures. Instruments employed: JASCO 410 (FTIR); JEOL JNM-LA 300 (NMR); Micromass Q-TOF AMPS MAX 10/6A (HRMS); Büchi Rotavapor R-200; VEEGO VMP-D (M.p.).

\section{General procedure}

Under dry $\mathrm{N}_{2}$ at $25{ }^{\circ} \mathrm{C}$, a stirred solution of $\mathrm{N}$-(thiobenzoyl)glycine $\left(\mathbf{1 b}, 1.0 \mathrm{mmol}\right.$ ) in dry $\mathrm{CH}_{2} \mathrm{Cl}_{2}$ $(3.0 \mathrm{ml})$ was treated dropwise with DCC $(1.2 \mathrm{mmol})$ in $\mathrm{CH}_{2} \mathrm{Cl}_{2}(2.0 \mathrm{ml}){ }^{5-7}$ After $10 \mathrm{~min}$., the mixture was treated with $\mathrm{Pb}(\mathrm{OAc})_{2} \cdot \mathrm{Pb}(\mathrm{OH})_{2}(0.05 \mathrm{mmol})$, followed by the aldehyde (3, $1.2 \mathrm{mmol}$ ). The resulting suspension was stirred for $20 \mathrm{~min}$., filtered through $\mathrm{Na}_{2} \mathrm{SO}_{4}$, and the filtrate distilled in vacuo. The resulting residue was column-chromatographed $\left(\mathrm{SiO}_{2}\right)$ eluting with EtOAc-hexane, to obtain the pure alkylidene or arylidene thioazlactones 5, characterized by IR $\left(v_{\max }\right),{ }^{1} \mathrm{H}$ NMR $\left(300 \mathrm{MHz}, \delta_{\mathrm{H}}\right),{ }^{13} \mathrm{C}$ NMR $\left(75 \mathrm{MHz}, \delta_{\mathrm{C}}\right)$ and high resolution mass (HRMS). The 
$\delta_{\mathrm{H}}$ and $\delta_{\mathrm{C}}$ are in $\mathrm{CDCl}_{3}$ relative to internal tetramethylsilane; $\delta_{\mathrm{C}}$ multiplicity was indicated by comparing the ${ }^{1} \mathrm{H}$ decoupled and DEPT spectra. Abbreviations: doublet (d), triplet (t), quartet (q), singlet (s); medium (m), strong (s), very strong (vs), weak (w).

The parent phenylthiazolone $\mathbf{2 b}$ could be prepared as above, but without adding $\mathrm{Pb}(\mathrm{OAc})_{2} \cdot \mathrm{Pb}(\mathrm{OH})_{2}$ and aldehyde 3 , and was characterized spectroscopically.

2-Phenylthiazol-5(4H)-one (2b). ${ }^{5} v_{\max } 3059$ (w), 1730 (vs, C=O), 1598 (m), 1448 (m), 1238 (w), $1048(\mathrm{~m}), 944$ (s), 765 (s) cm ${ }^{-1} ; \delta_{\mathrm{H}} 4.88$ (s, 2H), 7.45-7.57 (m, 3H, ArH), 7.80-7.84 (m, 2H, $\mathrm{Ar} H) ; \delta_{\mathrm{C}} 73.3\left(\mathrm{C}_{4}\right), 127.9,128.8,132.1,133.4,167.2\left(\mathrm{C}_{2}\right), 206.5\left(\mathrm{C}_{5}\right)$.

(4Z)-2-Phenyl-4-propylidenethiazol-5(4H)-one (5a). M.p. 51-52 ${ }^{\circ} \mathrm{C}$; $v_{\max } 2970$ (m), 1708 (vs, $\mathrm{C}=\mathrm{O}), 1628(\mathrm{~m}), 1528(\mathrm{~m}), 1447(\mathrm{w}), 1253(\mathrm{~s}), 1180(\mathrm{~m}), 975(\mathrm{~m}), 935(\mathrm{~m}), 766(\mathrm{~s}), 682(\mathrm{~s}) \mathrm{cm}^{-1} ; \delta_{\mathrm{H}} 1.21(\mathrm{t}$, 3H, $J$ 7.5 Hz, Me), 2.74-2.84 (m, 2H ), 6.68 (t, $1 \mathrm{H}, J 7.8 \mathrm{~Hz}, \mathrm{C}=\mathrm{CH}), 7.45-7.54$ (m, 3H, ArH), 7.92-7.95 (m, 2H, $\mathrm{Ar} H$ ); $\delta_{\mathrm{C}} 13.0$ (q, Me), 23.1 (t, $\mathrm{CH}_{2}$ ), 127.9 (d, $\mathrm{C}_{\mathrm{Ar}}$ ), 128.8 (d, $\mathrm{C}_{\mathrm{Ar}}$ ), $132.3\left(\mathrm{~d}, \mathrm{C}_{\mathrm{Ar}}\right), 133.3$ (s, $\mathrm{C}_{\mathrm{Ar}}$ ), 140.8 (d, C=CH-), $149.4\left(\mathrm{~s}, \mathrm{C}_{4}\right), 165.1\left(\mathrm{~s}, \mathrm{C}_{2}\right), 193.5$ (s, $\left.\mathrm{C}_{5}\right)$; HRMS: Found $(\mathrm{M}+\mathrm{H})$ 218.0630 [Calcd. $(\mathrm{M}+\mathrm{H})$ 218.0639].

(4Z)-4-Butylidene-2-phenylthiazol-5(4H)-one (5b). M.p. 51-53 ${ }^{\circ} \mathrm{C}$; $v_{\max } 2961$ (s), 1708 (vs, C=O), 1629 (s), 1597 (w), 1530 (s), 1447 (m), 1314 (w), 1255 (s), 968 (m), 765 (s) cm ${ }^{-1} ; \delta_{\mathrm{H}} 1.02$ (t, 3H, $J$ 7.5 Hz, Me), 1.57-1.69 (m, 2H, MeCH $\left.H_{2}\right), 2.71-2.79\left(\mathrm{~m}, 2 \mathrm{H}, \mathrm{C}=\mathrm{C}-\mathrm{CH}_{2}\right), 6.70(\mathrm{t}, 1 \mathrm{H}, J$ 8.1 Hz, C=CH-C), 7.44-7.56 (m, 3H, $\operatorname{Ar} H$ ), 7.89-7.96 (m, 2H, $\operatorname{Ar} H)$; $\delta_{\mathrm{C}} 13.9(\mathrm{q}, \mathrm{Me}), 21.9(\mathrm{t}$, $\mathrm{CH}_{2}$ ), $31.6\left(\mathrm{t}, \mathrm{CH}_{2}\right), 127.9\left(\mathrm{~d}, \mathrm{C}_{\mathrm{Ar}}\right), 128.8\left(\mathrm{~d}, \mathrm{C}_{\mathrm{Ar}}\right), 132.3$ (d, $\left.\mathrm{C}_{\mathrm{Ar}}\right), 133.3\left(\mathrm{~s}, \mathrm{C}_{\mathrm{Ar}}\right), 139.5$ (d, $\mathrm{C}=\mathrm{CH}-), 150.0$ (s, $\left.\mathrm{C}_{4}\right), 165.1$ (s, $\left.\mathrm{C}_{2}\right), 193.4\left(\mathrm{~s}, \mathrm{C}_{5}\right)$; HRMS: Found $(\mathrm{M}+\mathrm{H}) 232.0783$ [Calcd $(\mathrm{M}+\mathrm{H})$ 232.0796].

(4Z)-4-(2-Methylpropylidene)-2-phenylthiazol-5(4H)-one (5c). M.p. 50-51 ${ }^{\circ} \mathrm{C} ; v_{\max } 2963$ (m), 1732 (w, C=O), 1707(vs, C=O), 1630 (m), $1530(\mathrm{~m}), 1447$ (w), $1255(\mathrm{~m}), 1107(\mathrm{w}), 950(\mathrm{~m}) \mathrm{cm}^{-1} ; \delta_{\mathrm{H}} 1.20$ (d, $\left.6 \mathrm{H}, J 6.6 \mathrm{~Hz}, \mathrm{C}(\mathrm{Me})_{2}\right), 3.40-3.48(\mathrm{~m}, 1 \mathrm{H}, \mathrm{C}=\mathrm{C}-\mathrm{C} H), 6.52(\mathrm{~d}, 1 \mathrm{H}, J 9.9 \mathrm{~Hz}, \mathrm{C}=\mathrm{CH}), 7.44-7.56$ (m, 3H, $\operatorname{Ar} H$ ), 7.88-7.95 (m, 2H, $\operatorname{Ar} H$ ); $\delta_{\mathrm{C}} 22.0$ (q, Me), 29.1 (d, $\mathrm{Me}_{2} C$ ), 127.9 (d, $\mathrm{C}_{\mathrm{Ar}}$ ), 128.8 (d, $\mathrm{C}_{\mathrm{Ar}}$ ), 132.3 (d, $\mathrm{C}_{\mathrm{Ar}}$ ), 133.3 (s, $\mathrm{C}_{\mathrm{Ar}}$ ), 145.4 (d, C=CH-), 148.0 (s, $\left.\mathrm{C}_{4}\right), 164.9$ (s, $\left.\mathrm{C}_{2}\right), 193.9$ (s, $\mathrm{C}_{5}$ ); HRMS: Found $(\mathrm{M}+\mathrm{H}) 232.0804$ [Calcd. $(\mathrm{M}+\mathrm{H})$ 232.0796].

(4Z)-4-(2-Methylbutylidene)-2-phenylthiazol-5(4H)-one (5d). M.p. 55-57 ${ }^{\circ} \mathrm{C} ; v_{\max } 2957$ (m), 1710 (vs, C=O), 1629 (m), 1529 (m), 1447 (w), 1254 (m), 1163 (w), 975 (m), 765 (m) cm ${ }^{-1} ; \delta_{\mathrm{H}}$ 1.01 (d, 6H, $J 6.6 \mathrm{~Hz}, \mathrm{CMe}_{2}$ ), 1.89-1.96 (m, $1 \mathrm{H}, \mathrm{Me}_{2} \mathrm{CH}$ ), 2.68 ('t', $2 \mathrm{H}$, ' $J$ ' 7.8 Hz, C=C-CH $\mathrm{C}_{2}$ ), $6.72(\mathrm{t}, 1 \mathrm{H}, J 8.1 \mathrm{~Hz}, \mathrm{C}=\mathrm{C} H)$, 7.45-7.53 (m, 3H, $\operatorname{Ar} H), 7.92-7.95(\mathrm{~m}, 2 \mathrm{H}, \operatorname{Ar} H) ; \delta_{\mathrm{C}} 22.6(\mathrm{q}, \mathrm{Me})$, 28.5 (d, $\mathrm{Me}_{2} \mathrm{C}$ ), 38.4 (t, C=C-CH ), 127.9 (d, $\mathrm{C}_{\mathrm{Ar}}$ ), 128.8 (d, $\mathrm{C}_{\mathrm{Ar}}$ ), 132.3 (d, $\mathrm{C}_{\mathrm{Ar}}$ ), 133.3 (s, $\mathrm{C}_{\mathrm{Ar}}$ ), 138.7 (d, C=CH-), 150.5 (s, $\left.\mathrm{C}_{4}\right), 165.1$ (s, $\left.\mathrm{C}_{2}\right), 193.3$ (s, $\mathrm{C}_{5}$ ); HRMS: Found $(\mathrm{M}+\mathrm{H}) 246.0948$ [Calcd. $(\mathrm{M}+\mathrm{H})$ 246.0952].

(4Z)-4-Heptylidene-2-phenylthiazol-5(4H)-one (5e). (Viscous oil); $v_{\max } 2926$ (vs), 1709 (vs, C=O) $1629(\mathrm{~m}), 1529(\mathrm{~m}), 1447(\mathrm{w}), 1255$ (m), $971(\mathrm{w}), 765(\mathrm{~m}) \mathrm{cm}^{-1}$; $\delta_{\mathrm{H}} 0.89$ (t, 3H, $J 6.9 \mathrm{~Hz}$, Me), 1.25-1.42 (m, 6H, Me(CH $\left.)_{3}\right), 1.54-1.63\left(\mathrm{~m}, 2 \mathrm{H}, \mathrm{Me}\left(\mathrm{CH}_{2}\right)_{3} \mathrm{CH}_{2}\right), 2.72-2.80$ (m, 2H, C=C$\mathrm{CH}_{2}$ ), 6.69 (t, $1 \mathrm{H}, J$ 7.8 Hz, C=CH), 7.44-7.55 (m, 3H, $\left.\mathrm{Ar} H\right)$, 7.91-7.94 (m, 2H, $\left.\mathrm{Ar} H\right)$; $\delta_{\mathrm{C}} 14.0$ (q, Me), 22.5 (t, $\mathrm{CH}_{2}$ ), 28.5 (t, $\mathrm{CH}_{2}$ ), 29.0 (t, $\mathrm{CH}_{2}$ ), 29.6 (t, $\mathrm{CH}_{2}$ ), 31.5 (t, C=C-CH ), 127.9 (d, 
$\mathrm{C}_{\mathrm{Ar}}$ ), 128.7 (d, $\left.\mathrm{C}_{\mathrm{Ar}}\right), 132.3$ (d, $\mathrm{C}_{\mathrm{Ar}}$ ), 133.3 (s, $\mathrm{C}_{\mathrm{Ar}}$ ), 139.8 (d, C=CH-), $149.9\left(\mathrm{~s}, \mathrm{C}_{4}\right), 165.0$ (s, $\mathrm{C}_{2}$ ), 193.3 (s, C5); HRMS: Found (M+H) 274.1259 [Calcd. (M+H) 274.1265].

(4Z)-4-Benzylidene-2-phenylthiazol-5(4H)-one (5f) $)^{6,7}$ M.p. $130-131{ }^{\circ} \mathrm{C}$ (lit. $\left.{ }^{7} 130-132{ }^{\circ} \mathrm{C}\right) ; v_{\max }$ 3065 (w), 1698 (vs, C=O), 1611 (m), 1593 (m), 1485 (w), 1444 (m), 1255 (m), 986 (m), 932 (m), $752(\mathrm{~m}) \mathrm{cm}^{-1} ; \delta_{\mathrm{H}} 7.23(\mathrm{~s}, 1 \mathrm{H}, \mathrm{C}=\mathrm{CH}), 7.46-7.58(\mathrm{~m}, 6 \mathrm{H}, \operatorname{Ar} H)$, 7.99-8.02 (m, 2H, ArH), 8.24$8.27(\mathrm{~m}, 2 \mathrm{H}, \mathrm{ArH}) ; \delta_{\mathrm{C}} 128.2\left(\mathrm{~d}, \mathrm{C}_{\mathrm{Ar}}\right), 128.8\left(\mathrm{~d}, \mathrm{C}_{\mathrm{Ar}}\right), 128.9\left(\mathrm{~d}, \mathrm{C}_{\mathrm{Ar}}\right), 131.2\left(\mathrm{~d}, \mathrm{C}_{\mathrm{Ar}}\right), 131.3(\mathrm{~d}$, $\mathrm{C}_{\mathrm{Ar}}$ ), 132.6 (d, $\left.\mathrm{C}_{\mathrm{Ar}}\right), 133.1$ (d, C=CH-), 133.4 (s, $\left.\mathrm{C}_{\mathrm{Ar}}\right), 133.7$ (s, $\left.\mathrm{C}_{\mathrm{Ar}}\right), 146.1\left(\mathrm{~s}, \mathrm{C}_{4}\right), 166.7$ (s, $\left.\mathrm{C}_{2}\right)$, 194.5 (s, C5); HRMS: Found (M+H) 266.0640 [Calcd. (M+H) 266.0639].

(4Z)-2-Phenyl-4-((E)-3-phenylallylidene)thiazol-5(4H)-one (5g). M.p. 132-133 ${ }^{\circ} \mathrm{C}$; $v_{\max } 3054$ (w), 1692 (C=O), 1584 (s), 1446 (w), 1254 (m), 1129 (m), 974 (m), 745 (s) cm c $^{-1} \delta_{\mathrm{H}} 7.05-8.27$ $(\mathrm{m}) ; \delta_{\mathrm{C}} 124.6(\mathrm{~d},=C \mathrm{H}), 127.6(\mathrm{~d},=C \mathrm{H}), 128.0(\mathrm{~d}), 128.9(\mathrm{~d}), 128.95$ (d), $130.0(\mathrm{~d}), 132.4(\mathrm{~d})$, 132.6 (d), 133.5 (s), 136.1 (s), 145.0 (d, C $4=C H-), 147.3$ (s, C 4 ), 164.8 (s, C 2 ), 193.3 (s, C $)_{5}$ ); HRMS: Found $(\mathrm{M}+\mathrm{H}) 292.0798$ [Calcd. $(\mathrm{M}+\mathrm{H})$ 292.0796].

(4Z)-4-(4-Methoxybenzylidene)-2-phenylthiazol-5(4H)-one (5h). ${ }^{6}$ M.p. $158-159{ }^{\circ} \mathrm{C}$ (lit. ${ }^{6} 158-$ $\left.159{ }^{\circ} \mathrm{C}\right) ; v_{\max } 2936(\mathrm{w}), 1711(\mathrm{w}), 1675$ (s, C=O), 1595 (vs), 1566 (m), 1508 (m), 1307 (m), 1267 (vs), $1181(\mathrm{~m}), 1028(\mathrm{~m}), 992(\mathrm{~m}), 827(\mathrm{~m}) \mathrm{cm}^{-1}$; $\delta_{\mathrm{H}} 3.88$ (s, 3H, OMe), 6.99 (d, 2H, J 9.0 Hz, $\operatorname{Ar} H$ ), 7.21 (s, 1H, C=CH-), 7.47-7.56 (m, 3H, $\operatorname{ArH}$ ), 7.98-8.01 (m, 2H, ArH), 8.25 (d, 2H, J 9.0 $\mathrm{Hz}, \operatorname{Ar} H)$; $\delta_{\mathrm{C}} 55.4$ (q, OMe), 114.5 (d, $\mathrm{C}_{\mathrm{Ar}}$ ), 126.6 (s, $\mathrm{C}_{\mathrm{Ar}}$ ), $128.0\left(\mathrm{~d}, \mathrm{C}_{\mathrm{Ar}}\right), 128.8$ (d, $\left.\mathrm{C}_{\mathrm{Ar}}\right), 131.5$ (d, $\mathrm{C}_{\mathrm{Ar}}$ ), 132.2 (d, $\mathrm{C}_{\mathrm{Ar}}$ ), 133.5 (s, $\mathrm{C}_{\mathrm{Ar}}$ ), 135.4 (d, C=CH-), $144.4\left(\mathrm{~s}, \mathrm{C}_{4}\right), 162.3\left(\mathrm{~s}, \mathrm{C}_{\mathrm{Ar}}\right), 165.0$ (s, $\left.\mathrm{C}_{2}\right), 194.6$ (s, $\left.\mathrm{C}_{5}\right)$; HRMS: Found (M+H) 296.0741[Calcd. (M+H) 296.0745].

\section{Acknowledgements}

We thank CSIR (New Delhi) for generous fellowship support to M.S.

\section{References}

1. Chandrasekhar, S.; Karri, P. Tetrahedron Lett. 2007, 48, 785, and references cited therein.

2. Boaz, N. W.; Debenham, S. D.; Large, S. E.; Moore, M. K. Tetrahedron: Asymmetry 2003, 14, 3575.

3. Smith, M. B.; March, J. March's Advanced Organic Chemistry: Reactions, Mechanisms and Structure, 6th Edn.; Wiley: Hoboken (New Jersey), 2007; pp 61-63.

4. Barrett, G. C. Tetrahedron 1980, 36, 2023.

5. Bachi, M. D. J. Chem. Soc., Perkin Trans. 1 1972, 310.

6. Behringer, H.; Jepson, J. B. Chem. Ber. 1952, 85, 138.

7. Muxfeldt, H.; Behling, J.; Grethe, G.; Rogalski, W. J. Am. Chem. Soc. 1967, 89, 4991.

8. Kirchlechner, R.; Rogalski, W. Tetrahedron Lett. 1980, 21, 247. 
9. Oida, T.; Uemura, S. In Encyclopedia of Reagents for Organic Synthesis, 2nd Edn.; Paquette, L. A.; Crich, D.; Fuchs, P. L.; Molander, G. A. Eds.; Wiley: Chichester, 2009; Vol. 8, pp 5945-5946. 